\title{
Self-consistent theory of mobility edges in quasiperiodic chains
}

\author{
Alexander Duthie $\odot,{ }^{1, *}$ Sthitadhi Roy $\odot,{ }^{1,2, \dagger}$ and David E. Logan $\odot^{1,3, \ddagger}$ \\ ${ }^{1}$ Physical and Theoretical Chemistry, Oxford University, South Parks Road, Oxford OX1 3QZ, United Kingdom \\ ${ }^{2}$ Rudolf Peierls Centre for Theoretical Physics, Clarendon Laboratory, Oxford University, Parks Road, Oxford OX1 3PU, United Kingdom \\ ${ }^{3}$ Department of Physics, Indian Institute of Science, Bangalore 560012, India
}

(Received 14 December 2020; accepted 21 January 2021; published 1 February 2021)

\begin{abstract}
We introduce a self-consistent theory of mobility edges in nearest-neighbor tight-binding chains with quasiperiodic potentials. Demarcating boundaries between localized and extended states in the space of system parameters and energy, mobility edges are quite typical in quasiperiodic systems which lack the energyindependent self-duality of the commonly studied Aubry-André-Harper model. The potentials in such systems are strongly and infinite-range correlated, reflecting their deterministic nature and rendering the problem distinct from that of disordered systems. Importantly, the underlying theoretical framework introduced is model independent, thus allowing analytical extraction of mobility edge trajectories for arbitrary quasiperiodic systems. We exemplify the theory using two families of models and show the results to be in very good agreement with the exactly known mobility edges as well as numerical results obtained from exact diagonalization.
\end{abstract}

DOI: 10.1103/PhysRevB.103.L060201

The phenomenon of Anderson localization [1] is conventionally discussed in the context of disordered quantum systems. Quenched randomness is not, however, a prerequisite for localization. Indeed there exists a family of systemsthose with quasiperiodicity-which are nonrandom and deterministic yet host localization. The simplest and arguably most famous member of the family, the Aubry-André-Harper (AAH) model [2,3], hosts a localization transition [2] already in one dimension. Quasiperiodic chains also commonly show other interesting phenomena such as mobility edges, multifractal eigenstates both at and away from criticality, and "mixed phases" with both extended and localized eigenstates [4-16], and are readily implemented in experimental quantum emulators with ultracold atoms $[17,18]$.

While elusive in $d \leqslant 2$-dimensional, short-ranged disordered systems, mobility edges (ME) which demarcate the boundary between localized and extended states in the space of Hamiltonian parameters and energy are quite commonplace in quasiperiodic systems. The aforementioned AAH model is in effect a special case, where all eigenstates undergo a localization transition at a unique critical value of the quasiperiodic potential strength, and MEs cease to exist [2]. This is due to an exact duality in the model which is independent of energy. Any distortion of the model which breaks this duality typically leads to a genuine mobility edge in the spectrum.

Previous successes in understanding mobility edges in such systems have tended to focus on ideas specific to particular models, such as energy-dependent generalized duality transformations [8-10] or continuum models with bichromatic incommensurate potentials $[12,13]$. The propensity of

\footnotetext{
*alexander.duthie@chem.ox.ac.uk

${ }^{\dagger}$ sthitadhi.roy@chem.ox.ac.uk

¥david.logan@chem.ox.ac.uk
}

quasiperiodic models to possess MEs naturally means a theoretical framework to predict and understand them, which is model independent, is of basic importance, and this constitutes the central motivation of the present work.

We introduce a self-consistent theory of mobility edges in quasiperiodic systems based on the analysis of the local propagator, $G_{j}(t)=-i \Theta(t)\left\langle j\left|e^{-i H t}\right| j\right\rangle$, which physically measures the return probability amplitude of a state initialized at site $j$. The propagator is analyzed in the energy $(\omega)$ domain, wherein it acquires a self-energy whose imaginary part, $\Delta_{j}(\omega)$, is the central quantity of interest. Physically, $\Delta_{j}(\omega)$ is proportional to the rate of loss of probability from site $j$ into states of energy $\omega$ and is thus a natural diagnostic for localization or its absence. The characteristics of $\Delta_{j}(\omega)$ have in fact long proven successful in understanding Anderson transitions [1,19-24]. However, much of the analytical progress there was rendered possible by the independence of the random site energies and consequent independence of the local self-energies. Quasiperiodic systems in this regard present a significant challenge, as the deterministic nature of the potential means the site energies and self-energies are strongly and infinite-range correlated.

As concrete models for establishing and testing the theory, we consider one-dimensional nearest-neighbor tight-binding Hamiltonians of form

$$
H=V \sum_{j=0}^{L-1} \epsilon_{j} c_{j}^{\dagger} c_{j}+J \sum_{j=0}^{L-2}\left[c_{j}^{\dagger} c_{j+1}+\text { H.c. }\right],
$$

where $\epsilon_{j}$ encodes the quasiperiodic potential specific to the model (for specificity we consider $V, J \geqslant 0$ and unless stated otherwise take $J=1$ ). In particular, we consider two families of models, in which the quasiperiodic potential is generated via a periodic function with a period incommensurate with the underlying lattice. The first, referred to as the $\beta$ models 
$[\beta \in(-1,1)]$, is described by [10]

$$
\epsilon_{j}=\cos (2 \pi \kappa j+\phi)[1-\beta \cos (2 \pi \kappa j+\phi)]^{-1},
$$

with an irrational $\kappa$ (chosen as the golden mean) reflecting the quasiperiodicity and $\phi \in[0,2 \pi]$ a global phase shift used to accumulate statistics. The $\beta$ models are self-dual and host a single ME given by $\omega_{\mathrm{ME}}=(2-V) / \beta[10]$. Note that the standard AAH model is recovered as the $\beta=0$ limit, where the ME becomes a vertical line parallel to the $\omega$ axis at $V=2$, indicating that all states undergo a localization transition at $V=2$.

The second family is the so-called mosaic models [11] parametrized by an integer $l$. These non-self-dual models have an AAH potential on every $l$ th site, while all remaining sites have $\epsilon_{l}=0$. Formally,

$$
\epsilon_{j}= \begin{cases}\cos (2 \pi \kappa j+\phi) & : j=l k \\ 0 & : \text { otherwise }\end{cases}
$$

where $k \in \mathbb{Z}$. While MEs are known analytically for arbitrary $l$, for brevity we here consider explicitly the $l=2$ model, where the spectrum hosts two MEs given by $\omega_{\mathrm{ME}}= \pm 2 / \mathrm{V}$ [11].

Our theory centers on analysis of the local self-energy, $S_{j}(\omega)$, defined via the local Green function on site $j$ as

$$
G_{j}(\omega)=\left[\omega^{+}-V \epsilon_{j}-S_{j}(\omega)\right]^{-1},
$$

where $\omega^{+}=\omega+i \eta$ with $\eta=0^{+}$, and $S_{j}=X_{j}-i \Delta_{j}$. We focus on the imaginary part $\Delta_{j}=-\operatorname{Im} S_{j}$, since it serves as a probabilistic order parameter for a localization transition: $\Delta_{j}$ is (non)vanishing with unit probability in an (extended) localized phase [19]. For a one-dimensional nearest-neighbor model $S_{j}(\omega)$ can be expressed as

$$
S_{j}(\omega)=J^{2}\left[G_{j-1}^{(j)}(\omega)+G_{j+1}^{(j)}(\omega)\right],
$$

with $G_{j \pm 1}^{(j)}$ the local propagator for site $j \pm 1$ with site $j$ removed. As the local self-energy is a sum of two end-site propagators of a semi-infinite chain, localization or its absence can be inferred from the properties of end-site propagators alone [19]; we thus focus in the following on the self-energy of an end site, denoted $S_{0}(\omega)$. Since $S_{0}=J^{2} G_{1}^{(0)}$, and the imaginary part of the Green function is proportional to the local density of states (LDoS), the typical value of $\Delta_{0}$ (denoted henceforth as $\Delta_{\text {typ }}$ ) is proportional to the typical LDoS; indeed the latter was proposed as an order parameter for localization in both quasiperiodic [10] and also disordered systems [24-26]. This order parameter is finite in an extended phase and vanishes on approaching the transition from the extended side. In a localized phase by contrast, $\Delta_{0} \propto \eta$ vanishes. This leads to a corresponding (and considerably less studied) order parameter $y_{\text {typ }}^{-1}=\left(\Delta_{\text {typ }} / \eta\right)^{-1}$, which is finite in the localized phase and vanishes on approaching the transition from the localized side. A mobility edge is thus signalled by a simultaneous vanishing of $\Delta_{\text {typ }}$ from the extended side and divergence of $y_{\text {typ }}$ from the localized side.

In our theory we analyze both $\Delta_{\text {typ }}$ and $y_{\text {typ }}$. First, however, we show numerical results obtained from exact diagonalization for the two models Eqs. (2) and (3). The local propagator of the end site can be computed as $G_{0}(\omega)=$

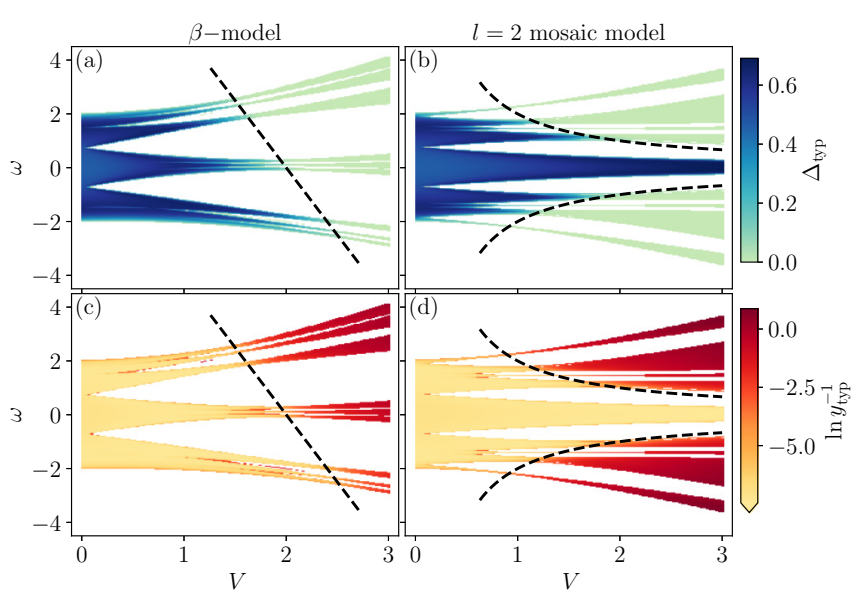

FIG. 1. Spectrum of the $\beta$-model (for $\beta=0.2$ ) and the $l=$ 2 mosaic model, color-coded with $\Delta_{\text {typ }}(\omega)$ (panels (a), (b)) and $\ln y_{\text {typ }}^{-1}(\omega)$ (panels (c), (d)). Black lines denote the known mobility edges $[10,11]$. In panels (a), (b) a finite $\Delta_{\text {typ }}$ indicates the presence of extended states and a vanishingly small value indicates localised states. Concomitantly, in panels (c), (d), a finite $y_{\text {typ }}^{-1}$ signals localised states whereas a vanishingly small $y_{\text {typ }}^{-1}$ (divergent $y_{\text {typ }}$ in the thermodynamic limit) signals extended states. These plots show $\Delta_{\text {typ }}$ to be a valid 'order-parameter' for the localisation-transition/mobilityedge coming from the extended side, while $y_{\text {typ }}^{-1}$ is its counterpart on approaching the transition from the localised side. Results obtained from exact diagonalisation with $L=2500$ sites and $\eta \propto 1 / L$, with averaging over $5000 \phi$-values.

$\left\langle 0\left|\left(\omega^{+}-H\right)^{-1}\right| 0\right\rangle$ with the matrix inversion performed numerically (where $|0\rangle$ denotes a state localized on the end site), from which $\Delta_{0}$ can be computed using Eq. (4). $\Delta_{\text {typ }}$ is then obtained as the geometric mean of the distribution of $\Delta_{0}$ by accumulating statistics over $\phi$. Since $\eta$ should be on the order of the mean level spacing we take $\eta=c / L$ (with $c \sim \mathcal{O}(1$ ) [27]), and $y_{\text {typ }}$ is simply obtained as $\Delta_{\text {typ }} / \eta$. The results are shown in Fig. 1, where the spectrum of both models is plotted in the $(V, \omega)$ plane with the data color coded according to $\Delta_{\text {typ }}$ in panels (a),(b) and $\ln y_{\text {typ }}^{-1}$ in (c),(d). The behavior of the numerically obtained $\Delta_{\text {typ }}$ and $y_{\text {typ }}^{-1}$-in particular their drop to vanishing values on approaching the MEs from the extended and localized sides, respectively - clearly shows their utility as order parameters. Having established that, we now turn to their theoretical analysis.

We begin by using Eqs. (4) and (5) to express $S_{0}(\omega)=$ $J^{2} G_{1}^{(0)}$ as a continued fraction (CF),

$$
S_{0}^{[n]}(\omega)=\frac{J^{2}}{\omega^{+}-V \epsilon_{1}-\frac{J^{2}}{\omega^{+}-V \epsilon_{2}-\frac{J^{2}}{\cdots-S_{n}^{[0]}(\omega)}}},
$$

where the superscript $[n]$ denotes that the $\mathrm{CF}$ has been continued (exactly) to order $n$ [28]. From this, $\Delta_{0}(\omega)$ and $y_{0}(\omega)$ can likewise be expressed as CFs.

At this stage, there are two conceptually distinct directions one can take. The first is to set the terminal self-energy in Eq. (6) to a typical value, $S_{n}^{[0]} \rightarrow-i \Delta_{\text {typ }}$, and obtain a distribution of $\Delta_{0}^{[n]}$ over an ensemble of $\phi$ values. This distribution 
depends parametrically on $\Delta_{\text {typ }}$. Self-consistency is then imposed by requiring that $\Delta_{\text {typ }}$ obtained from it is equal to the parametric $\Delta_{\text {typ }}$. This comprises a self-consistent mean-field theory at $n$th order, in the spirit of the self-consistent theory of Anderson localization [19]. The second, along the lines of Anderson's original work [1], is to analyze the convergence of the $\mathrm{CF}$ for $y_{0}$. This converges with unit probability in the localized phase, such that $y_{0}$ is finite; while in the extended phase it fails to converge, indicating a divergent $y_{0}$ in the thermodynamic limit. The convergence or lack thereof of the CF for $y_{0}$ is of course intimately connected to whether a finite or divergent self-consistent solution arises for $y_{\mathrm{typ}}$, which connects the two concepts [29].

In this work we take the first of these two directions and analyze the theory explicitly at leading order, $n=1$ (dropping the superscript $[n]$ from now). From Eq. (6),

$$
\Delta_{0}(\omega)=\frac{J^{2}\left(\eta+\Delta_{\mathrm{typ}}\right)}{\left(\omega-V \epsilon_{1}\right)^{2}+\left(\eta+\Delta_{\mathrm{typ}}\right)^{2}} .
$$

In a localized regime, where $\Delta_{\mathrm{typ}} \propto \eta$, the relevant quantity $y_{0}=\Delta_{0} / \eta$ can be expressed as

$$
y_{0}(\omega)=\frac{J^{2}\left(1+y_{\mathrm{typ}}\right)}{\left(\omega-V \epsilon_{1}\right)^{2}} .
$$

With $\langle\langle\cdots\rangle\rangle$ denoting an average over $\phi$ and end sites [30], $y_{\text {typ }}$ is self-consistently determined from $\ln y_{\mathrm{typ}}=\left\langle\left\langle\ln y_{0}\right\rangle\right\rangle$ and hence from Eq. (8) by

$$
\ln \left(1+y_{\text {typ }}^{-1}\right)=\left\langle\left\langle\ln \left(\omega-V \epsilon_{1}\right)^{2}\right\rangle\right\rangle-\ln J^{2} .
$$

In the extended regime, since $\Delta_{\text {typ }}$ is finite, the limit $\eta \rightarrow 0$ can be taken in Eq. (7), leading to $\Delta_{0}=J^{2} \Delta_{\text {typ }}\left[\left(\omega-V \epsilon_{1}\right)^{2}+\right.$ $\left.\Delta_{\text {typ }}^{2}\right]^{-1}$, which from $\ln \Delta_{\text {typ }}=\left\langle\left\langle\ln \Delta_{0}\right\rangle\right\rangle$ yields the desired equation for the self-consistent $\Delta_{\text {typ }}$,

$$
\left\langle\left\langle\ln \left[\left(\omega-V \epsilon_{1}\right)^{2}+\Delta_{\text {typ }}^{2}\right]\right\rangle\right\rangle-\ln J^{2}=0 .
$$

Before proceeding, we lay out clearly how the MEs (along with the regions of localized and extended states) are diagnosed using $y_{\text {typ }}$ and $\Delta_{\text {typ }}$.

At any point in the $(V, \omega)$ plane, a finite value of $\Delta_{\text {typ }}(V, \omega)$ implies that states there are extended. If by contrast $y_{\text {typ }}(V, \omega)=\Delta_{\text {typ }} / \eta$ is finite then states are localized, provided they exist for that $(V, \omega)$ point; which requires $\omega$ to lie within the localized band edges $\omega_{ \pm}^{(\mathrm{l})}$ of the average DoS, itself given to leading order by $\left\langle\left\langle\delta\left(\omega-V \epsilon_{1}\right)\right\rangle\right\rangle$ [31]. A point in the $(V, \omega)$ plane can thus be unambiguously identified as lying on a ME if (i) it lies in the interval $\omega \in\left[\omega_{-}^{(\mathrm{I})}, \omega_{+}^{(\mathrm{l})}\right]$ and (ii) $\Delta_{\text {typ }}$ and $y_{\text {typ }}$ simultaneously vanish and diverge (respectively) at that point. Additionally, the theory also predicts where localized and extended states may reside in the $(V, \omega)$ plane. Define $V_{-}\left(V_{+}\right)$as the $V$ where the MEs enter (leave) the spectrum on increasing $V$ from 0 , such that $V_{\mp}$ correspond to the points where $\omega_{\mathrm{ME}}=\omega_{ \pm}^{(1)}$ [31]. Then for $V<V_{-}$all states are extended, with spectral edges determined by the vanishing of $\Delta_{\text {typ }}(\omega)$. For $V>V_{+}$by contrast, all states are localized and the localized band edges form the spectral edges.

With this in hand, we turn to the results of our theory. Equations (9) and (10) are valid, respectively, on the localized and extended sides of a ME. Approaching the ME from the two sides amounts to $y_{\text {typ }}^{-1}$ and $\Delta_{\text {typ }}$ vanishing in Eqs. (9)
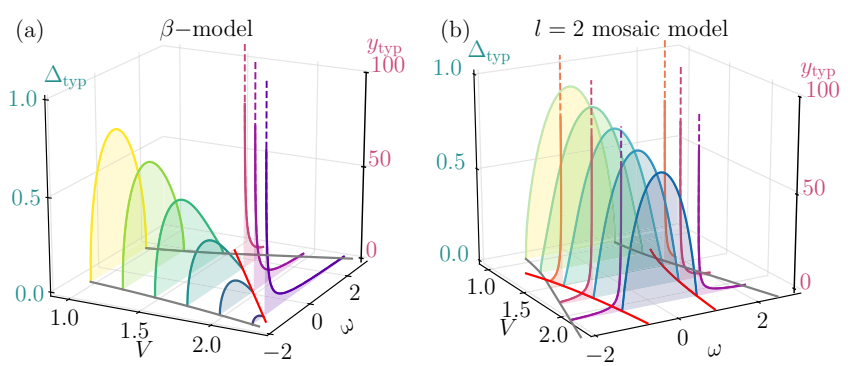

FIG. 2. Results for $\Delta_{\text {typ }}$ and $y_{\text {typ }}$ for (a) the $\beta$ model (with $\beta=$ 0.2 ) and (b) the $l=2$ mosaic model, obtained from the leading-order theory. A finite $\Delta_{\text {typ }}$ implies the presence of extended states, while its vanishing indicates that states if present are localized. Within the edges of the spectrum [shown as gray curves in the $(V, \omega)$ plane], the presence of localised states is indicated by a finite $y_{\mathrm{typ}}$. A simultaneous vanishing of $\Delta_{\text {typ }}(\omega)$ and divergence of $y_{\text {typ }}(\omega)$ signals the presence of mobility edges, which are denoted by the red curves in the $(V, \omega)$ plane. Note that the scales for $\Delta_{\text {typ }}$ and $y_{\text {typ }}$ in the plots are naturally very different.

and (10). Reassuringly, both these conditions yield identical expressions for the self-consistent ME,

$$
\left\langle\left\langle\ln \left[\left(\omega_{\mathrm{ME}}-V \epsilon_{1}\right)^{2}\right]\right\rangle\right\rangle-\ln J^{2}=0 .
$$

Significantly, this is completely independent of the specific model. Such a model-independent theory of MEs is a central result of this work. Moreover, this also shows that it is sufficient to analyze $y_{\text {typ }} \equiv y_{\text {typ }}(V, \omega)$ to obtain the MEs.

Turning to specific results, we first discuss the $\beta$ model. Equation (9) with $\epsilon_{j}$ given by Eq. (2) yields

$$
y_{\text {typ }}^{-1}=\left[(\omega \beta+V)^{2}-\lambda^{2}\right] / \lambda^{2},
$$

where $\lambda=1+\sqrt{1-\beta^{2}}$. Setting $y_{\text {typ }}^{-1}=0$ in Eq. (12) yields correctly a single [10] ME trajectory, given by

$$
\omega_{\mathrm{ME}}=\beta^{-1}\left[1+\sqrt{1-\beta^{2}}-V\right] \stackrel{\beta \ll 1}{\sim} \beta^{-1}(2-V) .
$$

Equation (13) is in qualitative agreement with the exactly known MEs [10] and asymptotically exact for $\beta \ll 1$; recovering as $\beta \rightarrow 0$ the AAH model result [2] that all states undergo a one-shot transition at the critical $V=2$. Additionally, from the average DoS we obtain the localized band edges as $\omega_{ \pm}^{(1)}=$ $\pm V /(1 \mp \beta)$, and hence via Eq. (13) that $V_{ \pm}=(1 \pm \beta) \lambda$. Combining these results shows that localized states reside in $\omega_{\mathrm{ME}}<\omega<\omega_{+}^{(1)}$ for $V \in\left(V_{-}, V_{+}\right)$and $\omega_{-}^{(\mathrm{l})}<\omega<\omega_{+}^{(1)}$ for $V>V_{+}$(where all states are localized). Analyzing Eq. (10) also shows that extended states exist in $\omega_{-}^{(\text {e) }}<\omega<\omega_{\mathrm{ME}}$ for $V \in\left(V_{-}, V_{+}\right)$and $\omega_{-}^{(\mathrm{e})}<\omega<\omega_{+}^{(\mathrm{e})}$ for $V \in\left(0, V_{-}\right)$(where all states are extended), with $\omega_{ \pm}^{(e)}$ the spectral edges obtained from the vanishing of $\Delta_{\text {typ }}(\omega)$ via Eq. (10), given by $\pm \beta^{2} \omega_{ \pm}^{(\mathrm{e})}=$ $\lambda \mp V \beta-\sqrt{\left(1-\beta^{2}\right) \lambda^{2} \mp 2 V \beta \lambda}$ (and evolving smoothly into $\omega_{ \pm}^{(1)}$ at $V=V_{ \pm}$). Thus the collective information of $\omega_{\mathrm{ME}}, \omega_{ \pm}^{(1 / \mathrm{e})}$, and $V_{ \pm}$maps out the entire localization phase diagram of the model in the $(V, \omega)$ plane. The above results, as well the resultant phase diagram of the model, are summarized in Fig. 2(a).

Turning to the $l=2$ mosaic models Eqs. (3) and (9) give

$$
y_{\mathrm{typ}}^{-1}=(V|\omega|-2) / 2 \text {, }
$$




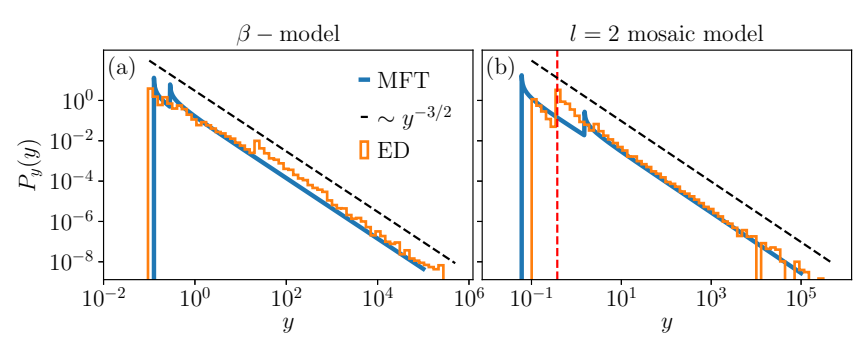

FIG. 3. Probability distributions of $y$ for the end site in the localized phase, for (a) the $\beta$ model with $\beta=0.2, V=3, \omega=0$ and (b) the $l=2$ mosaic model with $V=3$ and $\omega=2$. Blue lines show results from the leading-order theory. They are in excellent agreement with numerical data (shown in orange) obtained from exact diagonalization (ED) for $L=2500$ sites with statistics obtained over $5000 \phi$ values. The red dashed vertical line in (b) shows the $\delta$-function contribution, arising from even end sites [31]. The black dashed lines in both panels show a $y^{-3 / 2}$ tail, confirming that $P_{y}(y)$ has a characteristic Lévy tail.

with localized band edges $\omega_{ \pm}^{(1)}= \pm V$. From $y_{\text {typ }}^{-1}=0$ the model thus hosts two (symmetric in $\omega$ ) MEs given by

$$
\omega_{\mathrm{ME}, \pm}= \pm 2 / V
$$

which recovers precisely the exact result [11]. Note that the MEs never exit the spectrum, some states always being extended (such that $V_{+} \rightarrow \infty$ ), while the intersection of $\omega_{\mathrm{ME}, \pm}$ and $\omega_{ \pm}^{(1)}$ gives $V_{-}=\sqrt{2}$. Localized states thus arise for $V>V_{-}$ in the regimes $\omega_{-}^{(1)}<\omega<\omega_{\mathrm{ME},-}$ and $\omega_{\mathrm{ME},+}<\omega<\omega_{+}^{(1)}$. For $V<V_{-}$, the spectrum has solely extended states, with the band edges obtained from the vanishing of $\Delta_{\text {typ }}(\omega)$ [Eq. (10)] as $\omega_{ \pm}^{(\text {e) }}= \pm 2 / \sqrt{4-V^{2}}$. The model thus hosts extended states in the regime $\omega_{-}^{(\mathrm{e})}<\omega<\omega_{+}^{(\mathrm{e})}$ for $V<V_{-}$and $\omega_{\mathrm{ME},-}<\omega<$ $\omega_{\mathrm{ME},+}$ for $V>V_{-}$. Similarly to the $\beta$ model, these results, together with the resultant phase diagram in the $(V, \omega)$ plane, are summarized in Fig. 2(b). We add here that spectral gaps, indicative of self-similarity and seen in Fig. 1 for $\Delta_{\text {typ }}(\omega)$ and $y_{\text {typ }}(\omega)$, do not arise at the leading-order level discussed here but do emerge at higher order [29].

For each class of models, Eqs. (12) and (14) show that $y_{\text {typ }}$ diverges as $y_{\text {typ }} \sim\left(\omega-\omega_{\mathrm{ME}}\right)^{-1}$ on approaching the ME from the localized side [with $y_{\text {typ }} \sim(V-2)^{-1}$ as $V \rightarrow 2^{+}$in the $\omega$-independent AAH limit]. As this divergence is proportional to that of the localization length $\xi(\omega)$ [32], $\xi(\omega)$ thus diverges with a critical exponent of $v=1$, which likewise agrees with the exactly known Lyapunov exponents for the mosaic [11] and AAH [33] models.

Finally, while our natural focus has been on MEs, the theory also enables the self-consistent distributions of $y_{0}$ and
$\Delta_{0}$ to be obtained. Here we simply make some brief remarks about the distribution $P_{y}(y)$ of $y \equiv y_{0}$, which within the leading-order theory is given from Eq. (8) by

$$
P_{y}(y)=\left\langle\left\langle\delta\left(y-\frac{J^{2}\left(1+y_{\mathrm{typ}}\right)}{\left(\omega-V \epsilon_{1}(\phi)\right)^{2}}\right)\right\rangle\right\rangle .
$$

The analytic results for $P_{y}(y)$ [31] are rather unwieldy, so we show them graphically in Fig. 3 for representative $(V, \omega)$ points for the two models and compare them to results obtained from exact diagonalization, with which excellent agreement is seen. Two notable points to take away from the analytic expressions are, however, that (i) the distributions have a $\propto y^{-3 / 2}$ power-law (Lévy) tail and (ii) the support of the distributions have a sharp lower cutoff, which arises because the $\epsilon_{j}$ 's have a bounded distribution. We add that the Lévy tail in $P_{y}(y)$ seems quite universal, as it arises also in Anderson localization in disordered systems in the presence of both uncorrelated [19] as well as maximally correlated disorder [32].

In summary, we have introduced a self-consistent theory for MEs in quasiperiodic chains with nearest-neighbor hoppings. The theoretical framework is model independent, and its efficacy was demonstrated using two different classes of quasiperiodic models. The central object of interest is the imaginary part of the local self-energy, which acts as a probabilistic order parameter for a localization transition. MEs arising from the theory, and the localization phase diagrams in the space of Hamiltonian parameters and energy, were found to be in very good agreement with previous numerical and analytical results on the same families of models.

The present work suggests natural directions for further research. Here, the continued fraction Eq. (6) for $S_{0}(\omega)$ has been analyzed self-consistently upon truncating it at leading order. Generalizing the theory to arbitrarily high orders, analyzing the continued fraction's convergence, and connecting these approaches both conceptually and mathematically, forms the subject of a forthcoming work [29]. Using the $\omega$-dependent propagators extracted here to obtain analytical insights into some of the nonequilibrium dynamics of such systems in the time domain [34] is another interesting avenue for the future. Finally, developing a self-consistent theory of manybody localization in quasiperiodic systems on the Fock space, wherein the quasiperiodicity of the potentials will generate strong correlations in the Fock-space disorder [35,36], remains a challenge.

We thank I. Creed for helpful discussions. We also thank the EPSRC for support, under Grant No. EP/L015722/1 for the TMCS Centre for Doctoral Training, and Grant No. $\mathrm{EP} / \mathrm{S} 020527 / 1$.
[1] P. W. Anderson, Absence of diffusion in certain random lattices, Phys. Rev. 109, 1492 (1958).

[2] S. Aubry and G. André, Analyticity breaking and anderson localization in incommensurate lattices, Ann. Isr. Phys. Soc. 3, 18 (1980).

[3] P. G. Harper, Single band motion of conduction electrons in a uniform magnetic field, Proc. Phys. Soc. London, Sect. A 68, 874 (1955).
[4] R. E. Prange, D. R. Grempel, and S. Fishman, Wave functions at a mobility edge: An example of a singular continuous spectrum, Phys. Rev. B 28, 7370 (1983).

[5] S. Das Sarma, S. He, and X. C. Xie, Mobility Edge in a Model One-Dimensional Potential, Phys. Rev. Lett. 61, 2144 (1988).

[6] S. Das Sarma, S. He, and X. C. Xie, Localization, mobility edges, and metal-insulator transition in a class of 
one-dimensional slowly varying deterministic potentials, Phys. Rev. B 41, 5544 (1990).

[7] J. Biddle, B. Wang, D. J. Priour, and S. Das Sarma, Localization in one-dimensional incommensurate lattices beyond the aubryandré model, Phys. Rev. A 80, 021603(R) (2009).

[8] J. Biddle and S. Das Sarma, Predicted Mobility Edges in One-Dimensional Incommensurate Optical Lattices: An Exactly Solvable Model of Anderson Localization, Phys. Rev. Lett. 104, 070601 (2010).

[9] J. Biddle, D. J. Priour, B. Wang, and S. Das Sarma, Localization in one-dimensional lattices with non-nearest-neighbor hopping: Generalized anderson and aubry-andré models, Phys. Rev. B 83, 075105 (2011).

[10] S. Ganeshan, J. H. Pixley, and S. Das Sarma, Nearest Neighbor Tight Binding Models with an Exact Mobility Edge in One Dimension, Phys. Rev. Lett. 114, 146601 (2015).

[11] Y. Wang, X. Xia, L. Zhang, H. Yao, S. Chen, J. You, Q. Zhou, and X.-J. Liu, One-Dimensional Quasiperiodic Mosaic Lattice with Exact Mobility Edges, Phys. Rev. Lett. 125, 196604 (2020).

[12] D. J. Boers, B. Goedeke, D. Hinrichs, and M. Holthaus, Mobility edges in bichromatic optical lattices, Phys. Rev. A 75, 063404 (2007).

[13] X. Li, X. Li, and S. Das Sarma, Mobility edges in onedimensional bichromatic incommensurate potentials, Phys. Rev. B 96, 085119 (2017).

[14] S. Gopalakrishnan, Self-dual quasiperiodic systems with power-law hopping, Phys. Rev. B 96, 054202 (2017).

[15] S. Roy, I. M. Khaymovich, A. Das, and R. Moessner, Multifractality without fine-tuning in a Floquet quasiperiodic chain, SciPost Phys. 4, 25 (2018).

[16] H. Yao, H. Khoudli, L. Bresque, and L. Sanchez-Palencia, Critical Behavior and Fractality in Shallow One-Dimensional Quasiperiodic Potentials, Phys. Rev. Lett. 123, 070405 (2019).

[17] H. P. Lüschen, S. Scherg, T. Kohlert, M. Schreiber, P. Bordia, X. Li, S. Das Sarma, and I. Bloch, Single-Particle Mobility Edge in a One-Dimensional Quasiperiodic Optical Lattice, Phys. Rev. Lett. 120, 160404 (2018).

[18] F. A. An, K. Padavi, E. J. Meier, S. Hegde, S. Ganeshan, J. H. Pixley, S. Vishveshwara, and B. Gadway, Observation of tunable mobility edges in generalized Aubry-André lattices, arXiv:2007.01393.
[19] R. Abou-Chacra, D. J. Thouless, and P. W. Anderson, A selfconsistent theory of localization, J. Phys. C 6, 1734 (1973).

[20] E. N. Economou and M. H. Cohen, Existence of mobility edges in anderson's model for random lattices, Phys. Rev. B 5, 2931 (1972).

[21] D. J. Thouless, Electrons in disordered systems and the theory of localization, Phys. Rep. 13, 93 (1974).

[22] D. C. Licciardello and E. N. Economou, Study of localization in Anderson's model for random lattices, Phys. Rev. B 11, 3697 (1975).

[23] D. E. Logan and P. G. Wolynes, Anderson localization in topologically disordered systems, Phys. Rev. B 31, 2437 (1985).

[24] D. E. Logan and P. G. Wolynes, Dephasing and anderson localization in topologically disordered systems, Phys. Rev. B 36, 4135 (1987).

[25] M. Janssen, Statistics and scaling in disordered mesoscopic electron systems, Phys. Rep. 295, 1 (1998).

[26] V. Dobrosavljevi, A. A. Pastor, and B. K. Nikoli, Typical medium theory of Anderson localization: A local order parameter approach to strong-disorder effects, Europhys. Lett. 62, 76 (2003).

[27] Results are insensitive to the precise numerical prefactor.

[28] Equation (6) is formally the same as the CF running ad infinitum and thus remains exact.

[29] A. Duthie, S. Roy, and D. E. Logan (unpublished).

[30] The average over end sites is necessary to account for the different functional forms that end-site potentials may have, e.g., in the mosaic models [31].

[31] See Supplemental Material at http://link.aps.org/supplemental/ 10.1103/PhysRevB.103.L060201 for details of (i) the density of localized states and (ii) analytic results for the distribution $P_{y}(y)$ of $y \equiv \Delta_{0} / \eta$.

[32] S. Roy and D. E. Logan, Localization on Certain Graphs with Strongly Correlated Disorder, Phys. Rev. Lett. 125, 250402 (2020).

[33] D. J. Thouless, Bandwidths for a quasiperiodic tight-binding model, Phys. Rev. B 28, 4272 (1983).

[34] S. Roy, S. Mukerjee, and M. Kulkarni, Imbalance for a family of one-dimensional incommensurate models with mobility edges, arXiv:2010.09251.

[35] D. E. Logan and S. Welsh, Many-body localization in Fock space: A local perspective, Phys. Rev. B 99, 045131 (2019).

[36] S. Roy and D. E. Logan, Fock-space correlations and the origins of many-body localization, Phys. Rev. B 101, 134202 (2020). 